\title{
Kinetic Study of BLV Infectivity in BLV Susceptible and Resistant Cattle in Japan from 2017 to 2019
}

\author{
Lanlan Bai $^{1,2}{ }^{\mathbb{D}}$, Liushiqi Borjigin ${ }^{2}$, Hirotaka Sato ${ }^{2}$, Shin-Nosuke Takeshima ${ }^{1,2,3}{ }^{\mathbb{D}}$, Sakurako Asaji 4 (D), \\ Hiroshi Ishizaki ${ }^{5}$ D, Keiji Kawashima ${ }^{6}$, Yuko Obuchi ${ }^{7}$, Shinji Sunaga ${ }^{7}$, Asako Ando ${ }^{4,8}$, Hidehito Inoko ${ }^{4}$, \\ Satoshi Wada ${ }^{1}$ and Yoko Aida ${ }^{2,3,9, *}$
}

check for

updates

Citation: Bai, L.; Borjigin, L.; Sato, H.; Takeshima, S.-N.; Asaji, S.; Ishizaki, H.; Kawashima, K.; Obuchi, Y.; Sunaga, S.; Ando, A.; et al. Kinetic Study of BLV Infectivity in BLV Susceptible and Resistant Cattle in Japan from 2017 to 2019. Pathogens 2021, 10, 1281. https://doi.org/10.3390/ pathogens10101281

Academic Editor: Paul C. Bartlett

Received: 2 September 2021

Accepted: 2 October 2021

Published: 5 October 2021

Publisher's Note: MDPI stays neutral with regard to jurisdictional claims in published maps and institutional affiliations.

Copyright: (c) 2021 by the authors. Licensee MDPI, Basel, Switzerland. This article is an open access article distributed under the terms and conditions of the Creative Commons Attribution (CC BY) license (https:/ / creativecommons.org/licenses/by/ $4.0 /)$.
1 Photonics Control Technology Team, RIKEN Center for Advanced Photonics, 2-1 Hirosawa, Wako 351-0198, Saitama, Japan; lanlan.bai@riken.jp (L.B.); takesima@jumonji-u.ac.jp (S.-N.T.); swada@riken.jp (S.W.)

2 Viral Infectious Diseases Unit, RIKEN, 2-1 Hirosawa, Wako 351-0198, Saitama, Japan; liushiqi.borjigin@vetmed.hokudai.ac.jp (L.B.); hirosato@dokkyomed.ac.jp (H.S.)

3 Department of Food and Nutrition, Jumonji University, 2-1-28 Sugasawa, Niiza 352-8510, Saitama, Japan

4 GenoDive Pharma Inc., 4-14-1 Naka-cho, Atsugi 243-0018, Kanagawa, Japan; sasaji@genodive.co.jp (S.A.); aando@is.icc.u-tokai.ac.jp (A.A.); hinoko@genodive.co.jp (H.I.)

5 Grazing Animal Unit, Division of Grassland Farming, Institute of Livestock and Grassland Science, NARO, 768 Senbonmatsu, Nasushiobara 329-2793, Tochigi, Japan; hishizak@affrc.go.jp

6 Tobu and General Agricultural Office Livestock Hygiene Division, Ota 373-0805, Gunma, Japan; kawashima-ke@pref.gunma.lg.jp

7 Department of Agriculture Dairy and Livestock Division, Maebashi 371-8570, Gunma, Japan; obuchi-yu@pref.gunma.lg.jp (Y.O.); sunaga-shi@pref.gunma.lg.jp (S.S.)

8 Department of Molecular Life Science, Division of Basic Medical Science and Molecular Medicine, Tokai University School of Medicine, 143 Shimokasuya, Isehara 259-1119, Kanagasa, Japan

9 Laboratory of Global Infectious Diseases Control Science, Graduate School of Agricultural and Life Sciences, The University of Tokyo, 1-1-1 Yayoi, Bunkyo-ku, Tokyo 113-8657, Japan

* Correspondence: yoko-aida@g.ecc.u-tokyo.ac.jp; Tel.: +81-3-5841-5383

Abstract: Bovine leukemia virus (BLV) is the causative agent of enzootic bovine leukosis. Polymorphism in bovine lymphocyte antigen (BoLA)-DRB3 alleles is related to susceptibility to BLV proviral load (PVL), which is a useful index for estimating disease progression and transmission risk. However, whether differential BoLA-DRB3 affects BLV infectivity remains unknown. In a three-year follow-up investigation using a luminescence syncytium induction assay for evaluating BLV infectivity, we visualized and evaluated the kinetics of BLV infectivity in cattle with susceptible, resistant and neutral BoLA-DRB3 alleles which were selected from 179 cattle. Susceptible cattle showed stronger BLV infectivity than both resistant and neutral cattle. The order of intensity of BLV infectivity was as follows: susceptible cattle $>$ neutral cattle $>$ resistant cattle. BLV infectivity showed strong positive correlation with PVL at each testing point. BLV-infected susceptible cattle were found to be at higher risk of horizontal transmission, as they had strong infectivity and high PVL, whereas BLV-infected resistant cattle were low risk of BLV transmission owing to weak BLV infection and low PVL. Thus, this is the first study to demonstrate that the BoLA-DRB3 polymorphism is associated with BLV infection.

Keywords: BLV; syncytium formation; proviral load; BoLA-DRB3 allele; horizontal transmission; susceptible; resistant

\section{Introduction}

Bovine leukemia virus (BLV), an oncogenic member of the genus Deltaretrovirus of family Retroviridae, is the etiological agent of enzootic bovine leukosis (EBL), the most common neoplastic disease in cattle [1,2]. Most cattle infected with BLV are asymptomatic, and approximately one-third develop persistent lymphocytosis, characterized by nonmalignant polyclonal $\mathrm{CD}^{+} \mathrm{B}$-cell expansion, while only a small percentage develop EBL after 
a long latency period. Currently, vaccines or therapeutic procedures for preventing BLV transmission are lacking, because of which, the virus has spread worldwide. EBL was also added to the International Organization of Epizootics (OIE)-listed diseases, infections, and infestations in 2020 [3]. Currently, 40.9\% of dairy cows over 6 months of age and $28.7 \%$ of breeding cattle in Japan [4], $94.2 \%$ of dairy herds in America [5], 36.7\% of cattle and $78.3 \%$ of herds in Canada [6], over $81.8 \%$ of cattle and $99.1 \%$ of herds in Taiwan [7], over $50 \%$ of cattle and $86.8 \%$ of herds in Korea [8], approximately $31-41.9 \%$ of cattle in China are BLV seropositive [9,10]. BLV infection induces huge economic losses in cattle production and export [11] as it affects herd levels in high-performing dairy herds and cow longevity $[12,13]$. Reports show that BLV infection leads to significant economic losses in the dairy industry in the USA, which is estimated to be USD 525 million annually [11]. In addition, the economic loss per case of lymphosarcoma due to BLV was estimated to be USD 412 to USD 497 (CAD 635) in recent studies [13,14].

The free virus is unstable, and herd infection occurs mainly via BLV-infected cells. Horizontal transmission is recognized as the major route of BLV infection [15]. Either a small volume of blood with infected lymphocytes or a thousand infected cells are sufficient to infect a healthy animal [16]. BLV infection can be an iatrogenic disease, as it can be contracted during dehorning, ear tattooing, rectal palpation, reuse of needles and gloves $[17,18]$, and vectors $[19,20]$. In addition, insects and seasonal variations (the highest infection rates occur from July to September and the lowest infection rates occur during winter) are closely associated with the spread of BLV infection under natural conditions [20]. BLV can also infect newborns via the placenta during pregnancy and colostrum feeding from BLV-infected cows [21,22]. Binding of the BLV envelope to cationic amino acid transporter 1 [23], a cellular receptor of BLV, mediates cell fusion and virus entry. The BLV genome integrates into the host genome as a proviral load (PVL), which correlates strongly with disease progression [24,25] and BLV infectivity, assessed via syncytium formation [26]. Sato et al. developed a new assay called the luminescence syncytium induction assay (LuSIA), which is based on a new reporter cell line called CC81 (a feline cell line transformed by mouse sarcoma virus)-GREMG. This cell line can specifically respond to the expression of BLV regulatory protein, Tax, when cultured together with BLV-infected cells to form enhanced green fluorescent protein (EGFP)-expressing syncytium [26], which enables visualization of BLV cell-to-cell infection in vitro. Reports show that when PVLs exceed 10,000 copies $/ 10^{5}$ cells in blood, cattle secrete BLV in nasal secretions, saliva [27], and milk [28]. Furthermore, BLV from milk shows infectivity [28]. Therefore, BLV-infected cattle with high PVL are sources of infection for BLV-free cattle. Thus, PVL is considered a major diagnostic index for estimating BLV transmission risk [29].

PVL is strongly associated with the highly polymorphic bovine leukocyte antigen (BoLA)-DRB3 [30-32]. In total, 384 alleles are registered in the Immuno Polymorphism Database (IPD)-MHC database (https://www.ebi.ac.uk/ipd/mhc/group/BoLA/, accessed on 2 September 2021). The BoLA-DRB3*015:01 and DRB3*012:01 alleles are known susceptibility-associated markers related to high PVL. In contrast, the BoLA-DRB3*009:02, $D R B 3^{*} 014: 01: 01$ [30,31,33,34], and $D R B 3^{*} 002: 01$ alleles [33] are resistant markers associated with the development of high PVL. Other BoLA-DRB3 alleles were not significantly associated with PVL in vivo [30]. Thus, BoLA-DRB3 alleles are believed to determine cattlespecific differences resistant to BLV disease progression. However, whether polymorphisms in BoLA-DRB3 alleles are related to BLV infectivity is not known.

In present follow-up study spanning three years, we assessed whether different BoLA$D R B 3$ alleles were associated with BLV infectivity using white blood cells (WBCs) from BLV-infected cattle with different BoLA-DRB3 alleles and PVL.

\section{Results}

\subsection{Determination of BoLA-DRB3 Alleles and BLV Infection}

Previously, we had reported that $B o L A-D R B 3^{*} 015: 01$ and $D R B 3^{*} 012: 01$ alleles are associated with high PVL of BLV. The BoLA-DRB3*009:02, DRB3*014:01:01 and DRB3*002:01 
alleles were associated with high PVL resistance [30,31,33,34], while other BoLA-DRB3 alleles were not significantly associated with PVL in vivo [30]. For selecting BLV-infected cattle for three years (from 2017 to 2019) of follow-up investigation, we first collected blood from all cattle $(n=179)$ from Farm A in Tochigi prefecture, Japan, and analyzed the BoLA$D R B 3$ alleles and BLV infection. DNAs were extracted from blood samples to be typed $B o L A-D R B 3$ allele using sequence-based typing (SBT) method. Twenty alleles of BoLA$D R B 3$ in the IPD-MHC database were identified in our samples. As shown in Figure 1A, the results of allele frequency showed that $16.0 \%$ resistant alleles, $22.0 \%$ susceptible alleles, and $62.0 \%$ neutral alleles were detected in 179 cattle. The rate of resistant BoLA-DRB3 allele is known to be lesser than that of other alleles [31,35]. On the basis of the BoLA-DRB3 allele data, 179 cattle were grouped into three cattle groups: resistant cattle, susceptible cattle, and neutral cattle, which were defined to be carrying at least one susceptible, resistant or neutral allele in their genomes, respectively (Table 1).

A

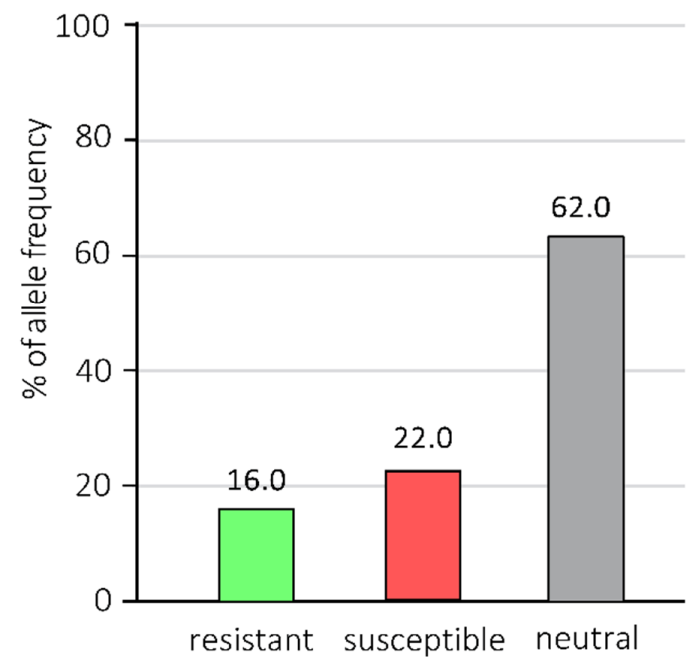

B

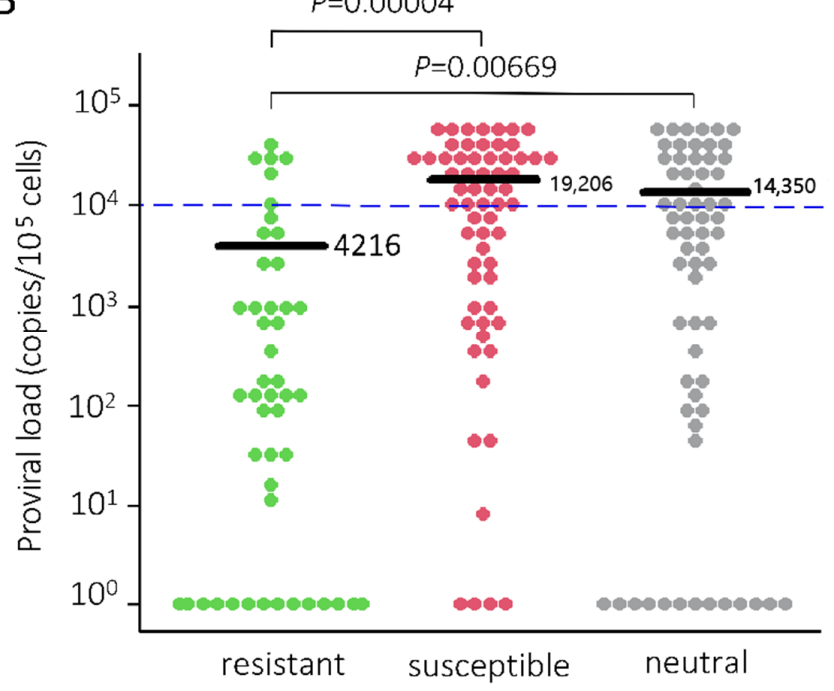

Figure 1. BoLA-DRB3 allele frequencies (A) and estimation of proviral loads (PVLs) (B). Blood samples were obtained from all 179 cattle and DNAs were extracted. BoLA-DRB3 alleles were typed using the SBT method, and the PVLs were measured using the CoCoMo-qPCR-2 method. All cattle were divided into resistant, susceptible, and neutral cattle groups based on the BoLA-DRB3 allele. The blue dotted line represents a BLV PVL of 10,000 copies $/ 10^{5}$ cells, which was set as the threshold between high and low PVL. The $X$-axis shows cattle classification, and the $Y$-axis shows percentage of allele frequency (A) and proviral loads (B). The mean PVL was compared among three groups and $p$ value was calculated using Tukey's test after the analysis of variance. $p<0.05$ represents statistically significant and $0.05<p<0.01$ represents statistically highly significant results.

Table 1. Distribution of cattle according to BoLA-DRB3 allele and PVL.

\begin{tabular}{ccccccc}
\hline \multirow{2}{*}{ Cattle Classification } & \multirow{2}{*}{ Cattle No. } & \multicolumn{2}{c}{ High PVL ${ }^{\mathbf{1}}$ Group } & \multicolumn{2}{c}{ Low PVL Group } & BLV-Free \\
\cline { 3 - 7 } & & BLV+ (\%) & Mean PVL & BLV+ (\%) & Mean PVL & BLV- (\%) \\
\hline Susceptible cattle $^{2}$ & 62 & $35(56.5 \%)$ & 32,218 & $23(37.1 \%)$ & 2746 & $4(6.4 \%)$ \\
Neutral cattle $^{3}$ & 68 & $26(38.2 \%)$ & 34,634 & $25(36.8 \%)$ & 3014 & $17(25.0 \%)$ \\
Resistant cattle $^{4}$ & 49 & $6(12.2 \%)$ & 28,688 & $27(55.1 \%)$ & 1275 & $16(32.7 \%)$ \\
Total & 179 & $67(37.4 \%)$ & 32,839 & $75(41.9 \%)$ & 2306 & $37(20.7 \%)$ \\
\hline
\end{tabular}

1. PVL: proviral load (copies $/ 10^{5}$ cells). ${ }^{2}$. Susceptible cattle carrying at least one susceptible BoLA-DRB3 allele in their genome. ${ }^{3 .}$ Resistant cattle carrying at least one resistant BoLA-DRB3 allele in their genome. ${ }^{4 .}$ Neutral cattle carrying other BoLA-DRB3 alleles.

Next, we measured the PVL of known and novel BLV variants in BLV-infected animals using BLV-CoCoMo-qPCR-2 (RIKEN Genesis, Kanagawa, Japan) [25,36-38]. In total, 142 out of 179 cattle $(79.3 \%)$ were positive and $37(20.7 \%)$ were negative for BLV PVL (Table 1$)$. 
The average PVL of each cattle group showed that the mean PVL of 49 resistant cattle was 4216 copies per $10^{5}$ cells, that of 62 susceptible cattle was 19,206 copies per $10^{5}$ cells, and that of 68 neutral cattle was 14,350 copies per $10^{5}$ cells (Figure 1B). Furthermore, the PVLs of resistant cattle were significantly higher than those of susceptible cattle $(p=0.00004)$ and neutral cattle ( $p=0.00669$ ) (Figure 1B). In contrast, 16 resistant cattle, 4 susceptible cattle, and 17 neutral cattle were BLV-negative (Table 1$)$.

In particular, the BLV provirus can be detected in milk, nasal mucus, and saliva samples of dairy cattle with PVL of more than 10,000, 14,000, and 18,000 copies $/ 10^{5}$ cells in blood samples, respectively $[27,28]$. Therefore, we set a threshold between high- and low-PVL to 10,000 copies $/ 10^{5}$ cells, as described previously [33]. Based on the threshold of PVL, 62 susceptible cattle, 68 neutral cattle, and 49 resistant cattle were divided into three groups: high PVL, low PVL, and BLV-free (Table 1). The range of PVL of 142 cattle was 14 to 70,870 copies per $10^{5}$ cells. The 35 susceptible cattle $(56.5 \%)$ had high PVL, with mean PVL of 32,218 copies $/ 10^{5}$ cells, ranging from 10,154 to 65,564 copies per $10^{5}$ cells, while the 23 susceptible cattle (37.1\%) had low PVL, with mean PVL of 2746 copies per $10^{5}$ cells, ranging from 9 to 9761 copies per $10^{5}$ cells. In contrast, only six resistant cattle had high PVL and the mean PVL was 28,688 copies $/ 10^{5}$ cells, ranging from 12,984 to 39,614 copies per $10^{5}$ cells, and the remaining 27 resistant cattle (55.1\%) had low PVL and the mean PVL was 1275 copies $/ 10^{5}$ cells, ranging from 14 to 8122 copies per $10^{5}$ cells (Table 1 ). In addition, as shown in Figure 1B, the PVLs in almost all resistant cattle were lower than the threshold $\left(10,000\right.$ copies $/ 10^{5}$ cells).

Taken together, the high infection rate in susceptible cattle with high PVL was compared to that in neutral and resistant cattle. Our results revealed the presence of BLVinfected resistant and susceptible cattle with different levels of PVLs, which were sufficient for the three-year follow-up study.

\subsection{Syncytium Formation Abilities in Cattle with Different BoLA-DRB3 Alleles}

PVL is a risk factor of BLV infection that correlates with BLV infectivity and is evaluated based on the syncytium formation assay $[25,26]$. Next, to select target BLV-infected cattle for the three-year follow-up investigation, we randomly tested 24 out of 142 cattle to analyze BLV infectivity using LuSIA [39] in the second half of 2017 (Table 2). Anti-BLV antibodies were detected in serum obtained from all tested blood samples. The $24 \mathrm{BLV}$ infected cattle contained 15 cattle carrying a susceptible BoLA-DRB3*015:01 or DRB3*012:01 allele, eight cattle carrying a resistant $B o L A-D R B 3^{*} 009: 02$ or $D R B 3^{*} 014: 01: 01$ allele, and one cattle carrying a neutral allele. The tested cattle with different levels of PVL were assessed using CoCoMo-qPCR-2. All susceptible cattle (S1-15) had high PVLs that exceeded 10,000 copies $/ 10^{5}$ cells, one neutral cattle N1 also carried 12,423 copies $/ 10^{5}$ cells, and all resistant cattle (R1-7) had low PVLs of less than 10,000 copies $/ 10^{5}$ cells. The WBCs obtained from the tested blood samples were co-cultured with the BLV reporter cell line CC81-GREMG, which can respond to Tax expression to express green fluorescence [39]. Nuclei were stained with Hoechst 33,342, which showed blue fluorescence. As shown in Figure 2A, susceptible cattle (S2, S3, and S7) had large syncytia that expressed EGFP, while resistant cattle (R2, R4, and R5) showed small number of syncytia. We compared the syncytium formation ability of all 24 tested cattle (Figure 2B). All resistant cattle, except R1 (8597 copies per $10^{5}$ cells), showed obviously weaker BLV infectivity than all susceptible cattle and one neutral cattle, as shown in Figure 2B. The results showed that BLV syncytium formation ability was weak in resistant cattle and strong in susceptible cattle.

Table 2. Information on BLV-infected cattle for assessing BLV infectivity and correlation analysis.

\begin{tabular}{|c|c|c|c|c|c|c|c|c|}
\hline \multirow{2}{*}{ Cattle No. } & \multirow{2}{*}{ Genotype } & \multicolumn{2}{|c|}{ BoLA-DRB3 } & \multirow{2}{*}{ PVL ${ }^{1,2}$} & \multirow{2}{*}{ gp51 Abs ${ }^{2}$} & \multirow{2}{*}{ Age $^{2}$ (Year) } & \multirow{2}{*}{$\begin{array}{c}\text { Syncytium Formation } \\
\text { Assay for Three-Year } \\
\text { Follow-Up Study }\end{array}$} & \multirow{2}{*}{$\begin{array}{c}\text { Correlation Analysis } \\
\text { of BLV Infectivity } \\
\text { with PVL }\end{array}$} \\
\hline & & A & B & & & & & \\
\hline S1 & Susceptible & 012:01 & 015:01 & 79,519 & +3 & 3.7 & $\mathrm{NT}^{4}$ & NT \\
\hline S2 & Susceptible & 001:01 & 015:01 & 35,758 & + & 7.8 & selected & Tested \\
\hline S3 & Susceptible & 011:01 & 015:01 & 37,707 & + & 6.2 & selected & Tested \\
\hline $\mathrm{S} 4$ & Susceptible & 011:01 & 015:01 & 10,780 & + & 3.9 & NT & NT \\
\hline S5 & Susceptible & 001:01 & 015:01 & 27,642 & + & 10.7 & NT & Tested \\
\hline S6 & Susceptible & 011:01 & 015:01 & 36,149 & + & 7.1 & NT & NT \\
\hline S7 & Susceptible & 001:01 & 015:01 & 39,024 & + & 11.2 & selected & Tested \\
\hline
\end{tabular}


Table 2. Cont.

\begin{tabular}{|c|c|c|c|c|c|c|c|c|}
\hline \multirow{2}{*}{ Cattle No. } & \multirow{2}{*}{ Genotype } & \multicolumn{2}{|c|}{ BoLA-DRB3 } & \multirow{2}{*}{ PVL ${ }^{1,2}$} & \multirow{2}{*}{ gp51 Abs ${ }^{2}$} & \multirow{2}{*}{ Age $^{2}$ (Year) } & \multirow{2}{*}{$\begin{array}{c}\text { Syncytium Formation } \\
\text { Assay for Three-Year } \\
\text { Follow-Up Study }\end{array}$} & \multirow{2}{*}{$\begin{array}{c}\text { Correlation Analysis } \\
\text { of BLV Infectivity } \\
\text { with PVL }\end{array}$} \\
\hline & & A & B & & & & & \\
\hline S8 & Susceptible & 001:01 & 015:01 & 17,981 & + & 5.8 & NT & NT \\
\hline S9 & Susceptible & 011:01 & 015:01 & 40,534 & + & 5.1 & NT & NT \\
\hline S10 & Susceptible & 015:01 & 015:01 & 18,062 & + & 6.8 & NT & Tested \\
\hline S11 & Susceptible & 015:01 & 018:01 & 7733 & + & 4.8 & NT & Tested \\
\hline S12 & Susceptible & 011:01 & 012:01 & 18,879 & + & 6.2 & NT & NT \\
\hline S13 & Susceptible & 010:01 & 015:01 & 12,097 & + & 1.4 & NT & NT \\
\hline S14 & Susceptible & 010:01 & 015:01 & 10,169 & + & 6.3 & NT & Tested \\
\hline S15 & Susceptible & 015:01 & 015:01 & 16,337 & + & 6.1 & NT & NT \\
\hline N1 & Neutral & 001:01 & 010:01 & 12,424 & NT & 0.5 & selected & Tested \\
\hline R1 & Resistant & 011:01 & 014:01:01 & 8597 & + & 6.2 & NT & Tested \\
\hline $\mathrm{R} 2$ & Resistant & 009:02 & 015:01 & 222 & + & 4.8 & selected & Tested \\
\hline R3 & Resistant & 001:01 & 014:01:01 & 165 & + & 10.8 & NT & NT \\
\hline $\mathrm{R} 4$ & Resistant & 014:01:01 & 014:01:01 & 0 & + & 6.2 & selected & Tested \\
\hline $\mathrm{R} 5$ & Resistant & 001:01 & 009:02 & 0 & + & 7.8 & selected & Tested \\
\hline R6 & Resistant & 011:01 & 014:01:01 & 54 & + & 5.4 & NT & NT \\
\hline R7 & Resistant & 011:01 & 014:01:01 & 468 & + & 5.1 & NT & Tested \\
\hline R8 & Resistant & 011:01 & 014:01:01 & 0 & + & 4.3 & NT & NT \\
\hline
\end{tabular}

1. PVL: proviral loads (copies $/ 10^{5}$ cells). ${ }^{2}$ Sampled to detect PVL and gp51 antibodies and calculate age in the second half of 2017.

3. Seropositive. ${ }^{4}$ NT: Not tested. Susceptible cattle carrying at least one susceptible BoLA-DRB3 allele in their genome. Resistant cattle carrying at least one resistant BoLA-DRB3 allele in their genome. Neutral cattle carrying other BoLA-DRB3 alleles.

\subsection{Assessing BLV Infectivity in Seven Selected Cattle in Three-Year Follow-Up Study}

In this three-year follow-up investigation, we would not constrain any decisions of the farmer as depositary breeding, selling, slaughtering, and eliminating et al. At the beginning, we decided to select 13 out of 24 cattle for BLV infectivity syncytium formation assay, but some selected cattle were sold out or deposited with other vendors' deposit breeding services, while at the time of our sampling. Therefore, as shown in Table 2, only 7 out of 13 cattle were sampled at each checking time owing to some selected cattle were sold out (S10 sold out after the second time sampled) or deposited with other vendors' deposit breeding services, while at the time of our sampling. These $7 \mathrm{BVL}$-infected cattle contained three susceptible cattle (S2, S3, and S7), three resistant cattle (R2, R4, and R5), and one cattle with the neutral allele, N1. From October 2017 to August 2019, we collected blood samples from targeted cattle seven times and assessed BLV infectivity using LuSIA [39]. The syncytium was formed via cell fusion after BLV infection, and hence syncytium formation ability reflected BLV infectivity. The WBCs were isolated from each blood sample and syncytium formation assay was performed using the BLV reporter cell line CC81-GREMG at each time point. The permanently BLV-infected FLK-BLV cell line was used as a positive control. EGFP-expressing syncytia were observed using an EVOS2 fluorescence microscope. The syncytia index (SI) of syncytium indicated the relative syncytium number of each sample when syncytium number in FLK-BLV cells was set to 1. As shown in Figure 3, the SI of syncytium in resistant cattle R2, R4, and R5 was consistently lower than that in susceptible cattle $\mathrm{S} 2, \mathrm{~S} 3$, and $\mathrm{S} 7$ at each checking point in the follow-up period, indicating that BLV infectivity was weak in resistant cattle but strong in susceptible cattle. The neutral cattle N1 also showed medium BLV infectivity in the three cattle groups. Taken together, the results showed that resistant cattle had weak BLV infectivity, which did not increase throughout the long post-infection period. In contrast, susceptible cattle showed strong BLV infection during their infectious phase. Our results indicated that resistant cattle indeed have low risk of BLV transmission, and that selection of these resistant cattle represents a promising approach for controlling the spread of the virus. 
A

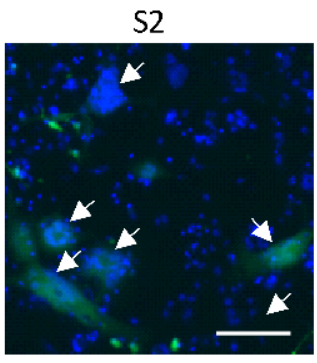

R2

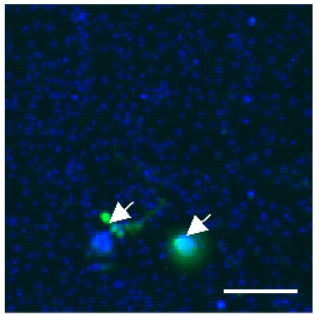

N1

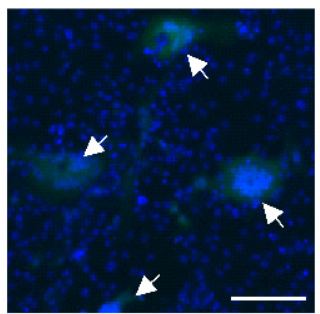

B

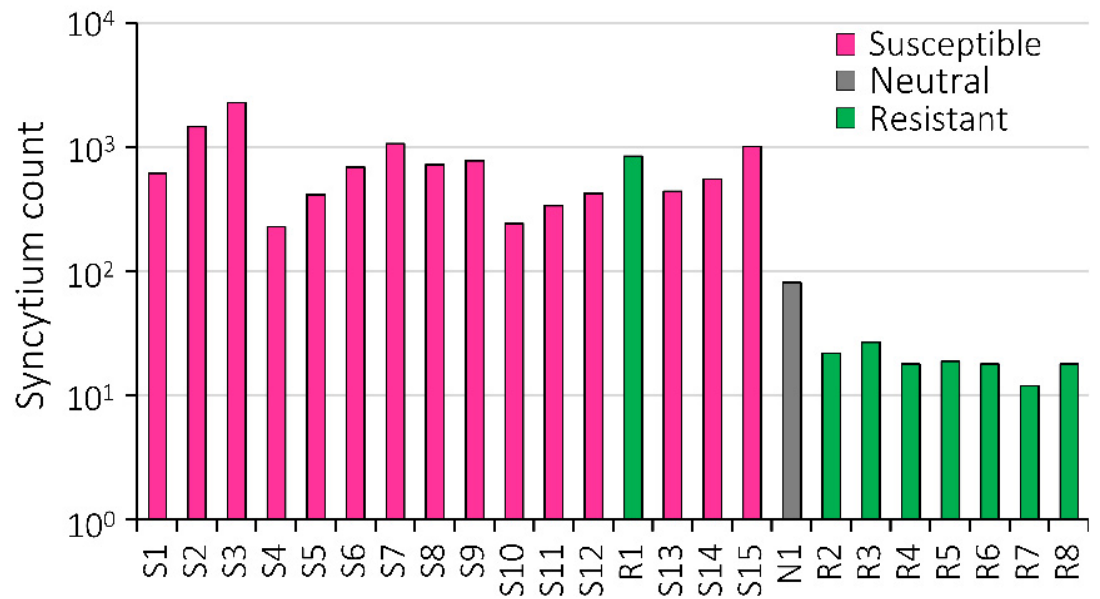

Figure 2. BLV infectivity of white blood cells (WBCs) from BLV-susceptible, -resistant, and -neutral cattle. (A) Syncytium formation. (B) Comparison of syncytium formation ability. WBCs were isolated from blood of BLV-infected susceptible cattle $(S ; n=15)$, resistant cattle $(\mathrm{R} ; n=8)$, and one neutral cattle $(\mathrm{N})$, and then co-cultured with the BLV reporter cell line, CC81-GREMG, which responded to BLV Tax expression for five days to form an EGFP-expressing syncytium. The fluorescent syncytia were observed using an EVOS2 fluorescence microscope. BLV-infected fetal lamb kidney (FLK-BLV) cells permanently infected with BLV were used as the positive control. Mock-treated cells were used as the negative control. White arrows show EGFP-expressing syncytia in (A). The $Y$-axis shows syncytium count, and the $X$-axis shows cattle number. 


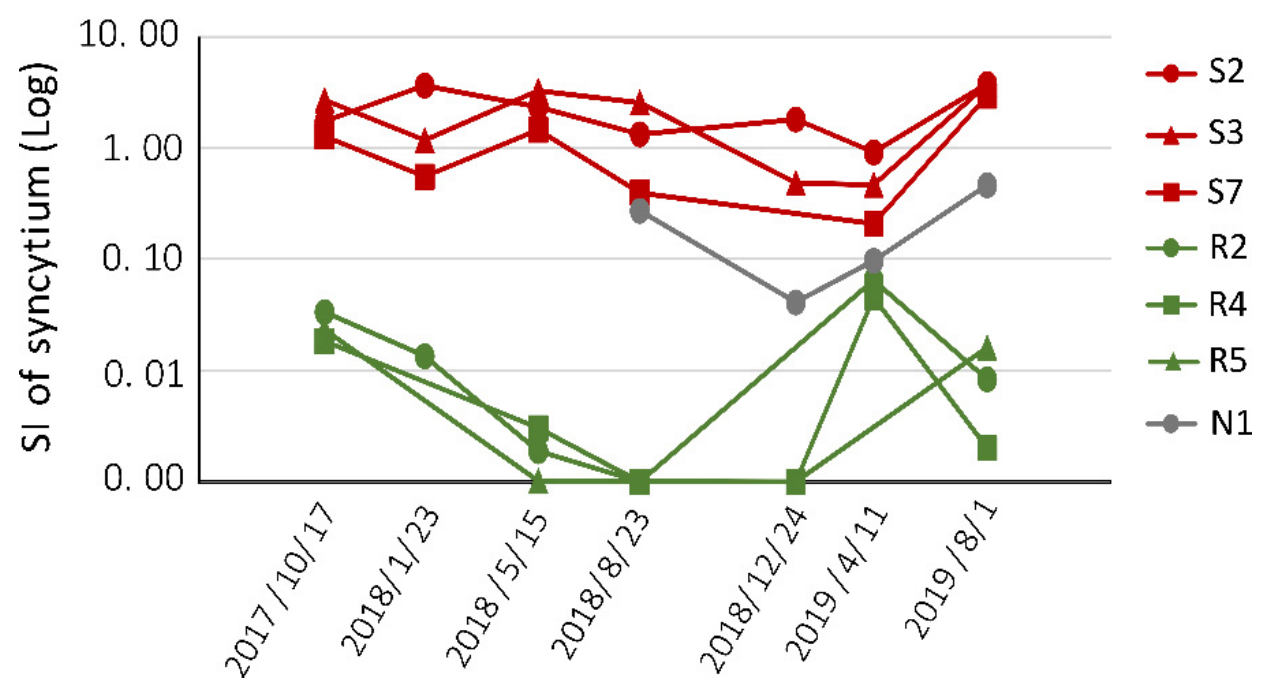

Figure 3. Kinetics of BLV infectivity in BLV-susceptible, -resistant, and -neutral cattle. White blood cells (WBCs) were isolated from the blood of BLV-infected BLV-susceptible (red), -resistant cattle (green), and neutral cattle (grey), and then co-cultured with the BLV reporter cell line, CC81-GREMG, for five days. The fluorescent syncytia were observed using an EVOS2 fluorescence microscope. Permanently infected FLK-BLV cells were used as the positive control. The SI of syncytium was calculated from the proportion of syncytium number that was formed in sample WBCs, while the positive control was set at one.

\subsection{Correlation of BLV Infectivity and PVL in Three-Year Follow-Up Study}

BoLA-DRB3 alleles are associated with PVL in vivo. In addition, our three-year followup study clearly showed that BLV infectivity was weak in resistant cattle, strong in susceptible cattle, and medium in neutral cattle N1. However, the association of the BoLA-DRB3 allele with BLV syncytium formation ability is unknown. Therefore, to clarify the relationship between BoLA-DRB3 allele and BLV infectious ability, we increased the number of tested cattle to 13, which the selected cattle in the depositary breeding service returned to the farm (Table 2), and then evaluated BLV infectivity using LuSIA and PVL using BLV-CoCoMo-qPCR-2 seven times during our follow-up study. We considered the effects of uncontrollable external factors on the infectivity of BLV and PVL at each sampling time; thus, we analyzed the data detected at each checking time for separate analyses. The PVL and number of syncytia of tested cattle at each checking point were represented seven times, as shown in Figure 4A. With the exception of sample R1, the susceptible cattle showed higher PVLs (blue spot) and larger number of syncytia (orange bar) than those in neutral cattle (gray bar) and resistant cattle (green bar). Results of previous reports [24,33], have shown that the BoLA-DRB3*009:02 allele suppressed PVL better than the $B o L A-D R B 3^{*} 014: 01: 01$ allele. Here, the resistant cattle R1 carried the BoLA-DRB3*014:01:01 allele, and had high PVL and a large number of syncytia. Furthermore, to analyze the correlation between PVL and syncytium at each checking point, we constructed a scatter graph and performed linear regression analysis using the data at each time point for separate analyses. Interestingly, a strong positive correlation was observed, and the Spearman's rank correlation coefficient $(R)$ ranged from 0.7611 to $0.9350(R=0.8537 \pm 0.0716)$ (Figure 4B). This is also consistent with our previous results [39]. Taken together, our findings demonstrated that resistant cattle showed lower PVL and weaker BLV infectivity in their long infectious period than susceptible cattle. 
A
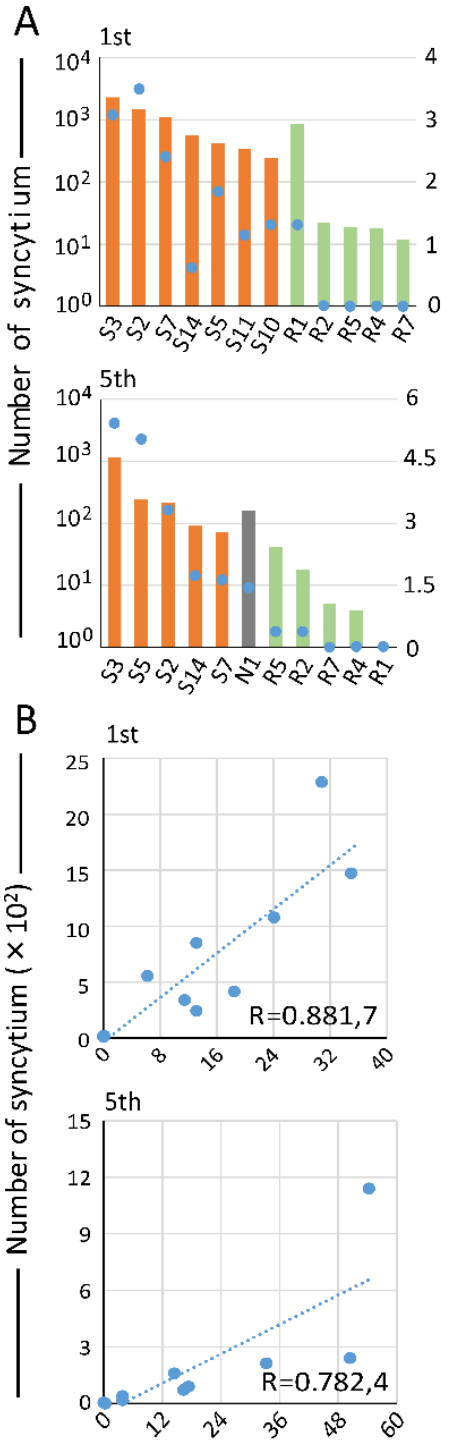
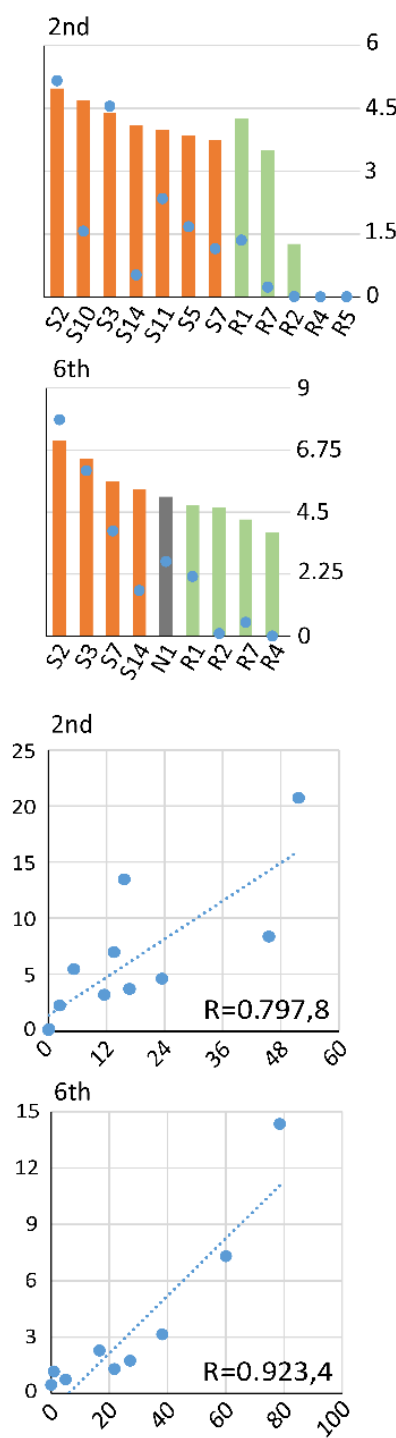

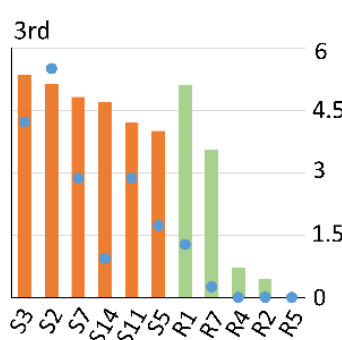

7 th 8
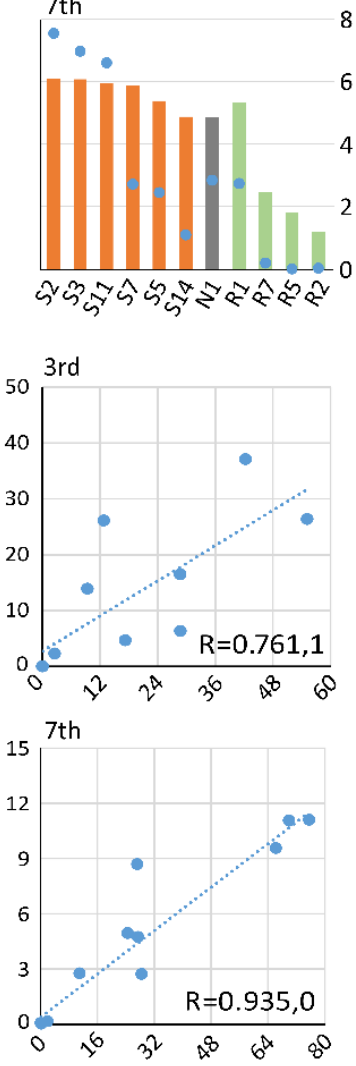

- Susceptible cattle

- Neutral cattle

- Resistant cattle

- Proviral load
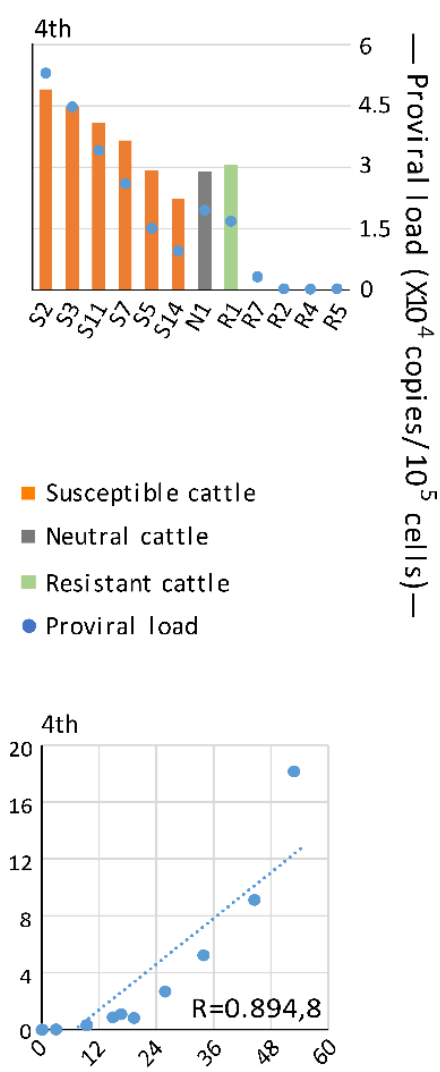

Proviral load ( $\times 10^{3}$ copies $/ 10^{5}$ cells)

Figure 4. Correlation between BLV infectivity and proviral loads (PVLs) in followed-up cattle. (A) Syncytium number and proviral load of tested cattle at each checking point. (B). Correlation between syncytium formation ability and PVL at each checking point. During blood sampling, DNAs were extracted and the PVLs were measured using CoCoMo-qPCR-2. The syncytium detected in the white blood cells of BLV-infected cattle were co-cultured with CC81-GREMG cells for five days. Fluorescent syncytia were observed using an EVOS2 fluorescence microscope. BLV infectivity was indicted by the number of syncytia in the bar graph. The bold line represents the approximate curve $(R=$ correlation coefficient).

\section{Discussion}

Among the many risk factors associated with BLV infection, BoLA complex polymorphisms are one of the most important host factors strongly involved in controlling the subclinical progression of BLV infection by regulating PVL in vivo $[24,27,28,33,40-44]$, as PVL is an indicator of disease progression. Several studies have shown that BoLA-DRB3 is a highly polymorphic gene that affects susceptibility to BLV-induced B cell lymphoma [45,46], and PVL [31]. BLV infection mainly occurs via cell-to-cell transmission in individuals and herds. Based on the results of a three-year follow-up study, we collected blood from seven BLV-infected cattle, including three susceptible cattle (BoLA-DRB3*015:01 or DRB3*012:01), three resistant cattle (BoLA-DRB3*009:02 or DRB3 $\left.{ }^{*} 014: 01: 01\right)$, and one neutral cattle, and showed for the first time that BoLA-DRB3 alleles are associated with BLV infectivity. 
$B o L A-D R B 3^{*} 016: 01$ and $D R B 3^{*} 015: 01$ have been reported to be associated with high PVL in Japanese Black and Holstein cattle, respectively [30,45]. All BLV-susceptible cattle tested also had high PVLs, and WBCs from their blood showed strong BLV infectivity in a follow-up investigation from 2017 to 2019. In contrast, BoLA-DRB3*009:02 is known to play an important immunological role in suppressing viral replication, resulting in resistance to disease progression in both Japanese Black and Holstein cattle [47]. Cattle R2 and R5 carry resistant BoLA-DRB3*009:02 allele, and they had weak BLV infectivity and low PVL in the long-infected phase. In addition, no BLV transmission was observed over 30 months of contact when cattle carrying resistant BoLA-DRB3*009:02 allele with low PVL were incorporated into a BLV-negative dairy herd. The $B o L A-D R B 3^{*} 0902$ cattle with low PVL disrupted the BLV transmission chain [29]. Our results are consistent with previously reported that BLV-infected susceptible cattle have high PVL and remain at its high level, and resistant cattle keep low PVL over a long infection period $[33,48]$. Thus, resistant cattle be considered that are low risk of BLV transmission.

The BoLA-DRB3*014:01:01 has also been reported to suppress both BLV PVL and lymphoma [33]. The tested resistant cattle, with the exception of cattle R1, showed lower PVL and weaker BLV infectivity than susceptible cattle in three years. In the initial phase of the follow-up study, resistant cattle R1 had higher PVL and infectivity than the other tested resistant cattle, despite carrying the $B o L A-D R B 3^{*} 014: 01: 01$ allele. Although $B o L A-$ $D R B 3^{*} 014: 01: 01$ suppressed PVL to some extent in vivo, infection suppression was possibly not completely achieved. The PVL of R1 increased and exceeded the threshold value of 10,000 copies $/ 10^{5}$ cells. Besides, the BoLA-DRB3*002:01 allele [33] has been reported that is a resistant marker associated with high PVL. The nearly $20 \%$ (6 of 33) BLV-infected resistant cattle had high PVL carry $D R B 3^{*} 014: 01: 01$ allele or $D R B 3^{*} 002: 01$ allele. Thus, it is considered that resistant $D R B 3^{*} 009: 02$ allele can strongly suppress the development of high PVL than $D R B 3^{*} 014: 01: 01$ allele or $D R B 3^{*} 002: 01$ allele. It may be that $D R B 3^{*} 009: 02$ combines with other host factors to thoroughly suppress high PVL.

In Japanese Black herds, the distribution of allele frequencies for $B o L A-D R B 3^{*} 016: 01$ was over $30 \%$, $D R B 3^{*} 009: 02$ was $2.0 \sim 5.8 \%$, and $D R B 3^{*} 014: 01: 01$ was $1.4 \sim 2.5 \%$. In Holstein cattle herds, the distribution of allele frequencies for $B o L A-D R B 3 * 015: 01$ was , $\%$, $D R B 3^{*} 009: 02$ was 5.2 7.1\%, DRB3* 014:01:01 was 2.4 7.1\%, and $D R B 3^{*} 002: 01$ was $4.5 \sim 8.6 \%$ [45]. The distribution of BoLA-DRB3 allele frequencies in our selected Farm $\mathrm{A}$ also was in the above distribution ranges. Thus, Farm A can be a representative of Japanese farms, and it can interpret Japanese farms. Besides, a strong positive correlation was observed between BLV infectivity and PVL. Therefore, our results are considered that can reflect the state of the entire farm.

$\mathrm{R}$ cattle have lower PVL and weak BLV infectivity than susceptible cattle, indicating that $\mathrm{R}$ cattle are low risk of BLV transmission. The amount of PVL increased or changed over time. Resistant and susceptible cattle with the same amount of PVL were not detected in the tested cattle at any time point. Therefore, it was difficult to directly compare the direct association of the BoLA-DRB3 allele with BLV infectivity. However, two major hypotheses were considered. One was that the resistant allele was involved in eliminating BLV infections to reduce its original integration; therefore, PVL did not increase. The other was that resistant alleles regulate viral replication, which lowers PVL production, resulting in weaker BLV infectivity than in susceptible cattle. The resistant BoLA-DRB3 allele directly or indirectly affects the BLV infectivity to reduce BLV transmission. In addition, the levels of anti-gp51 antibodies differ significantly in resistant and susceptible cattle [33], indicating that BoLA MHC Class II molecules present different antigen epitopes for T and B cells in these cattle to activate the adaptive and humoral immune response. Resistant cattle may have strong immunity and can protect from BLV.

Although some resistant cattle are infected with BLV, they maintain low levels of PVL due to their long infectious period. Furthermore, a considerable proportion of resistant cattle are BLV-negative. In contrast, most susceptible cattle are easily infected with BLV and have high PVLs in the short post-infection period. Previous reports have shown that 
the BLV provirus may be detected in milk, nasal mucus, and saliva of dairy cattle with PVLs $>10,000,14,000$, and 18,000 copies per $10^{5}$ cells in blood samples, respectively [28]. In addition, BLV from raw milk is infectious [28]. Our tested susceptible cattle, with the exception of S14, had high PVL, which was more than the threshold value of 10,000 copies $/ 10^{5}$ cells. The range of PVL in S14 was also 5274-16,532 copies $/ 10^{5}$ cells. Therefore, we believe that BLV-infected susceptible cattle might produce BLV in their secretions and milk. BLVinfected susceptible cattle were found to have a significantly higher risk of horizontal transmission. Similarly, vertical transmission risk and horizontal transmission appeared to be extremely high for dams and cattle with susceptible alleles compared to those with resistant alleles [49]. In contrast, the resistant BoLA-DRB3 allele can effectively inhibit or prevent BLV infection, as observed in this study. Thus, BLV-infected resistant cattle are low risk of BLV transmission because of weak BLV infection with low PVL. In addition, PVL was maintained at low level in most dams with resistant alleles, thereby reducing the risk of vertical BLV transmission [49]. In contrast, BLV-infected susceptible cattle are at a high risk of BLV infectivity. Therefore, in addition to eliminate and removal BLV transmission in high-risk susceptible cattle, breeding of low-risk resistant cattle is considered a promising strategy to gradually reduce the infection rate while minimizing economic loss. Furthermore, based on our understanding of the evolution of the BoLA-DRB3 allele in cattle, the development of breeding strategies aimed at improving resistance to infectious diseases and designing of broadly effective vaccines against susceptible cattle should be considered in the future.

\section{Materials and Methods}

\subsection{Blood Sample Collection}

In Japan, the number of cattle raised per farm is 93.9. We selected an entire herd of 179 cattle containing Holstein cattle, Japanese Black, and F1 hybrids, which is about twice as large as the average scale. Blood samples were collected from 179 cattle of Farm A in Tochigi Prefecture, Japan, and stored in ethylenediaminetetraacetic acid (EDTA). Serum was collected to detect BLV antibodies. This study was approved by the animal ethical committee, and the animal care and use RIKEN animal experiments committee (Approval Number H29-2-104) and the animal care committee of the Institute of Livestock and Grassland Science, NARO (Approval Number: 1711B082, 1811B084, 1911B041).

\subsection{DNA Extraction}

DNAs were extracted using the Wizard ${ }^{\circledR}$ Genomic DNA Purification Kit (Promega corporation, Madison, WI, USA) at each time point from the blood samples according to the manufacturer's instructions.

\subsection{Detection of BLV Proviral Load}

BLV PVL was measured in all DNA samples from blood using the BLV-CoCoMo-qPCR2 method (RIKEN Genesis, Kanagawa, Japan) as described previously [24,25,37,38,50,51].

\subsection{BoLA-DRB3 Allele Typing}

$B O L A-D R B 3$ alleles were genotyped using the polymerase chain reaction (PCR)sequence-based typing (SBT) method as described previously [52]. Briefly, primers $\mathrm{F}$ (5'-CGCTCCTGTGAYCAGATCTATCC- $\left.3^{\prime}\right)$ and R (5'-CACCCCCGCGCTCACC-3') were used for CDNA amplification of BoLA-DRB3 exon 2 using PCR. The PCR products were purified to sequence using the Big Dye Terminator v1.1 Cycle Sequencing Kit. The sequence conditions were as follows: 25 cycles at $96^{\circ} \mathrm{C}$ for $10 \mathrm{~s}, 50{ }^{\circ} \mathrm{C}$ for $5 \mathrm{~s}$, and $60^{\circ} \mathrm{C}$ for $2 \mathrm{~min}$. Sequence data were analyzed using ASSIGN 400 ATF software (Conexio Genomics, Fremantle, Australia) to identify the BoLA-DRB3 alleles. Susceptible cattle identified as carrying at least one susceptible $B o L A-D R B 3$ allele, resistant cattle carrying at least one resistant $B o L A-D R B 3$ allele, and neutral cattle carrying other BoLA-DRB3 alleles in their genome. 


\subsection{Measurement of gp51 Antibodies}

Anti-BLV gp51 antibodies in all serum samples were measured using an anti-BLV antibody ELISA kit (JNC, Tokyo, Japan), according to the manufacturer's instructions.

\subsection{Detection of Syncytium Formation}

The blood samples were treated with red blood cell lysis buffer (Abbott Diagnostics Technologies AS, Oslo, Norway) to remove red blood cells, and the pellets were washed with cold phosphate-buffered saline (PBS) and then resuspended in Dulbecco's modified Eagle's medium (DMEM) (Thermo Fisher Scientific, Waltham, MA, USA) with $10 \%$ fetal bovine serum (FBS; Sigma-Aldrich, St. Louis, MO, USA) [53]. WBCs ( $1 \times 10^{5}$ cells/well) were applied to the BLV reporter cell lines, CC81-GREMG (5 × 104 cells/well), [23,26,39,54] that were pre-cultured for one day in a 12-well plate. After 3 days of incubation, the plate was washed with PBS, and fresh DMEM with $10 \%$ FBS was added and incubated for up to $48 \mathrm{~h}$. The cells were washed with PBS and fixed with PBS containing $3.7 \%$ formaldehyde and $10 \mathrm{mg} / \mathrm{mL}$ Hoechst 33,342 (Millipore Sigma). The fixed cells were observed for fluorescent-positive syncytia using an EVOSFL Auto 2 Cell Imaging System (Thermo Fisher Scientific). The permanently BLV-infected FLK-BLV cell line $[55,56]$ was used as a positive control for this assay.

\subsection{Statistical Analysis}

The correlation coefficient (R) between the syncytium and PVL was calculated using Excel with the Pearson function. Analysis of variance followed by Tukey's' post-hoc test was used to determine the significance of the means of PVL for multiple comparisons. Differences were considered to be significant at $p<0.05$, and strongly significant at $p<0.01$ and $p<0.001$.

\section{Conclusions}

We measured BLV infectivity in WBCs via syncytium formation assay among susceptible, resistant, and neutral cattle at three years of follow-up. The susceptible cattle carrying the BoLA-DRB3*015:01 or DRB3*012:01 allele showed strong infectivity and high PVL in their blood. Resistant cattle carrying the BoLA-DRB3*009:02 or DRB3*014:01:01 allele maintained weaker BLV infectivity and lower PVL at each checking point than the susceptible and neutral cattle. Although we did not directly compare BLV infectivity in the same amount of PVL of susceptible cattle and resistant cattle in vitro, the BoLA-DRB3 allele was found to be directly or indirectly associated with BLV infectivity. Consequently, breed selection based on resistant BoLA-DRB3 allele is an effective strategy for reducing and controlling BLV infection.

Author Contributions: Conceptualization, Y.A. and L.B. (Lanlan Bai); sample collection, L.B. (Lanlan Bai), L.B. (Liushiqi Borjigin), H.S., S.-N.T., H.I. (Hiroshi Ishizaki), K.K., Y.O., S.S. and Y.A.; methodology, L.B. (Lanlan Bai) and Y.A.; software, L.B. (Lanlan Bai); validation, L.B. (Lanlan Bai), H.S., S.A. and L.B. (Liushiqi Borjigin); formal analysis, L.B. (Lanlan Bai); investigation, L.B. (Lanlan Bai), L.B. (Liushiqi Borjigin); resources, A.A., H.I. (Hidehito Inoko), S.W. and Y.A.; data curation, L.B. (Lanlan Bai); writing-original draft preparation, L.B. (Lanlan Bai); writing—review and editing, L.B. (Lanlan Bai) and Y.A.; visualization, L.B. (Lanlan Bai); supervision, Y.A.; project administration, Y.A.; funding acquisition, Y.A. All authors have read and agreed to the published version of the manuscript.

Funding: This work was supported by Grants-in-Aid for Scientific Research (A (16H02590)) from the Japan Society for the Promotion of Science (JSPS), by a grant from the Project of the NARO Bio-oriented Technology Research Advancement Institution (the special scheme project on vitalizing management entities of agriculture, forestry and fisheries; Grant No. 16930548), and by a grant from the program for the promotion of Basic and Applied Research for Innovations in Bio-oriented Industry (9151615). 
Institutional Review Board Statement: This study was approved by the animal ethical committee, and the animal care and use RIKEN animal experiments committee (Approval Number H29-2-104) and the animal care committee of the Institute of Livestock and Grassland Science, NARO (Approval Number: 1711B082, 1811B084, 1911B041).

Informed Consent Statement: Not applicable.

Data Availability Statement: The data presented in this study are available on request from the corresponding author.

Acknowledgments: We are also grateful to all members of the Viral Infectious Diseases Unit, RIKEN for help and helpful suggestions.

Conflicts of Interest: The authors declare no conflict of interest.

\section{References}

1. Aida, Y.; Murakami, H.; Takahashi, M.; Takeshima, S.N. Mechanisms of pathogenesis induced by bovine leukemia virus as a model for human T-cell leukemia virus. Front. Microbiol. 2013, 4, 328. [CrossRef] [PubMed]

2. Gillet, N.; Florins, A.; Boxus, M.; Burteau, C.; Nigro, A.; Vandermeers, F.; Balon, H.; Bouzar, A.B.; Defoiche, J.; Burny, A.; et al. Mechanisms of leukemogenesis induced by bovine leukemia virus: Prospects for novel anti-retroviral therapies in human. Retrovirology 2007, 4, 18. [CrossRef]

3. Enzootic Bovine Leukosis. OIE World Animal Health Information Database (WAHID Interface). 2019; pp. 1113-1124. Available online: https:/ / www.oie.int/fileadmin/Home/eng/Health_standards/tahm/3.04.09_EBL.pdf (accessed on 10 August 2021).

4. Murakami, K.; Kobayashi, S.; Konishi, M.; Kameyama, K.; Tsutsui, T. Nationwide survey of bovine leukemia virus infection among dairy and beef breeding cattle in Japan from 2009-2011. J. Vet. Med. Sci. 2013, 75, 1123-1126. [CrossRef]

5. LaDronka, R.M.; Ainsworth, S.; Wilkins, M.J.; Norby, B.; Byrem, T.M.; Bartlett, P.C. Prevalence of Bovine Leukemia Virus Antibodies in US Dairy Cattle. Vet. Med. Int. 2018, 2018, 5831278. [CrossRef]

6. Nekouei, O.; VanLeeuwen, J.; Sanchez, J.; Kelton, D.; Tiwari, A.; Keefe, G. Herd-level risk factors for infection with bovine leukemia virus in Canadian dairy herds. Prev. Vet. Med. 2015, 119, 105-113. [CrossRef] [PubMed]

7. Hsieh, J.C.; Li, C.Y.; Hsu, W.L.; Chuang, S.T. Molecular Epidemiological and Serological Studies of Bovine Leukemia Virus in Taiwan Dairy Cattle. Front. Vet. Sci. 2019, 6, 427. [CrossRef] [PubMed]

8. Suh, G.H.; Lee, J.C.; Lee, C.Y.; Hur, T.Y.; Son, D.S.; Ahn, B.S.; Kim, N.C.; Lee, C.G. Establishment of a bovine leukemia virus-free dairy herd in Korea. J. Vet. Sci. 2005, 6, 227-230. [CrossRef] [PubMed]

9. Yang, Y.; Fan, W.; Mao, Y.; Yang, Z.; Lu, G.; Zhang, R.; Zhang, H.; Szeto, C.; Wang, C. Bovine leukemia virus infection in cattle of China: Association with reduced milk production and increased somatic cell score. J. Dairy Sci. 2016, 99, 3688-3697. [CrossRef] [PubMed]

10. Yu, C.; Wang, X.; Zhou, Y.; Wang, Y.; Zhang, X.; Zheng, Y. Genotyping bovine leukemia virus in dairy cattle of Heilongjiang, northeastern China. BMC Vet. Res. 2019, 15, 179. [CrossRef]

11. Ott, S.L.; Johnson, R.; Wells, S.J. Association between bovine-leukosis virus seroprevalence and herd-level productivity on US dairy farms. Prev. Vet. Med. 2003, 61, 249-262. [CrossRef]

12. Erskine, R.J.; Bartlett, P.C.; Byrem, T.M.; Render, C.L.; Febvay, C.; Houseman, J.T. Association between bovine leukemia virus, production, and population age in Michigan dairy herds. J. Dairy Sci. 2012, 95, 727-734. [CrossRef] [PubMed]

13. Rhodes, J.K.; Pelzer, K.D.; Johnson, Y.J.; Russek-Cohen, E. Comparison of culling rates among dairy cows grouped on the basis of serologic status for bovine leukemia virus. J. Am. Vet. Med. Assoc. 2003, 223, 229-231. [CrossRef] [PubMed]

14. Alessa, K.; Henk, H.; Karin, O.; Robert, W.; Jada, T.; Eldon, S.; Frank, V.D.M. Economic evaluation of 4 bovine leukemia virus control strategies for Alberta dairy farms. J. Dairy Sci. 2019, 102, 2578-2592. [CrossRef]

15. Mekata, H.; Sekiguchi, S.; Konnai, S.; Kirino, Y.; Horii, Y.; Norimine, J. Horizontal transmission and phylogenetic analysis of bovine leukemia virus in two districts of Miyazaki, Japan. J. Vet. Med. Sci. 2015, 77, 1115-1120. [CrossRef] [PubMed]

16. Dimmock, C.K.; Chung, Y.S.; MacKenzie, A.R. Factors affecting the natural transmission of bovine leukaemia virus infection in Queensland dairy herds. Aust. Vet. J. 1991, 68, 230-233. [CrossRef] [PubMed]

17. Hopkins, S.G.; DiGiacomo, R.F. Natural transmission of bovine leukemia virus in dairy and beef cattle. Vet. Clin. N. Am. Food Anim. Pract. 1997, 13, 107-128. [CrossRef]

18. Kohara, J.; Konnai, S.; Onuma, M. Experimental transmission of Bovine leukemia virus in cattle via rectal palpation. Jpn. J. Vet. Res. 2006, 54, 25-30. [PubMed]

19. Kohara, J.; Takeuchi, M.; Hirano, Y.; Sakurai, Y.; Takahashi, T. Vector control efficacy of fly nets on preventing bovine leukemia virus transmission. J. Vet. Med. Sci. 2018, 80, 1524-1527. [CrossRef] [PubMed]

20. Manet, G.; Guilbert, X.; Roux, A.; Vuillaume, A.; Parodi, A.L. Natural mode of horizontal transmission of bovine leukemia virus (BLV): The potential role of tabanids (Tabanus spp.). Vet. Immunol. Immunopathol. 1989, 22, 255-263. [CrossRef]

21. Meas, S.; Usui, T.; Ohashi, K.; Sugimoto, C.; Onuma, M. Vertical transmission of bovine leukemia virus and bovine immunodeficiency virus in dairy cattle herds. Vet. Microbiol. 2002, 84, 275-282. [CrossRef] 
22. Agresti, A.; Ponti, W.; Rocchi, M.; Meneveri, R.; Marozzi, A.; Cavalleri, D.; Peri, E.; Poli, G.; Ginelli, E. Use of polymerase chain reaction to diagnose bovine leukemia virus infection in calves at birth. Am. J. Vet. Res. 1993, 54, 373-378.

23. Bai, L.; Sato, H.; Kubo, Y.; Wada, S.; Aida, Y. CAT1/SLC7A1 acts as a cellular receptor for bovine leukemia virus infection. FASEB J. 2019, fj201901528R. [CrossRef]

24. Ohno, A.; Takeshima, S.N.; Matsumoto, Y.; Aida, Y. Risk factors associated with increased bovine leukemia virus proviral load in infected cattle in Japan from 2012 to 2014. Virus Res. 2015, 210, 283-290. [CrossRef]

25. Jimba, M.; Takeshima, S.N.; Murakami, H.; Kohara, J.; Kobayashi, N.; Matsuhashi, T.; Ohmori, T.; Nunoya, T.; Aida, Y. BLVCoCoMo-qPCR: A useful tool for evaluating bovine leukemia virus infection status. BMC Vet. Res. 2012, 8, 167. [CrossRef] [PubMed]

26. Sato, H.; Watanuki, S.; Murakami, H.; Sato, R.; Ishizaki, H.; Aida, Y. Development of a luminescence syncytium induction assay (LuSIA) for easily detecting and quantitatively measuring bovine leukemia virus infection. Arch. Virol. 2018, 163, 1519-1530. [CrossRef] [PubMed]

27. Yuan, Y.; Kitamura-Muramatsu, Y.; Saito, S.; Ishizaki, H.; Nakano, M.; Haga, S.; Matoba, K.; Ohno, A.; Murakami, H.; Takeshima, S.N.; et al. Detection of the BLV provirus from nasal secretion and saliva samples using BLV-CoCoMo-qPCR-2: Comparison with blood samples from the same cattle. Virus Res. 2015, 210, 248-254. [CrossRef] [PubMed]

28. Watanuki, S.; Takeshima, S.N.; Borjigin, L.; Sato, H.; Bai, L.; Murakami, H.; Sato, R.; Ishizaki, H.; Matsumoto, Y.; Aida, Y. Visualizing bovine leukemia virus (BLV)-infected cells and measuring BLV proviral loads in the milk of BLV seropositive dams. Vet. Res. 2019, 50, 102. [CrossRef]

29. Juliarena, M.A.; Barrios, C.N.; Ceriani, M.C.; Esteban, E.N. Hot topic: Bovine leukemia virus (BLV)-infected cows with low proviral load are not a source of infection for BLV-free cattle. J. Dairy Sci. 2016, 99, 4586-4589. [CrossRef]

30. Juliarena, M.A.; Poli, M.; Sala, L.; Ceriani, C.; Gutierrez, S.; Dolcini, G.; Rodriguez, E.M.; Marino, B.; Rodriguez-Dubra, C.; Esteban, E.N. Association of BLV infection profiles with alleles of the BoLA-DRB3.2 gene. Anim. Genet. 2008, 39, 432-438. [CrossRef]

31. Takeshima, S.N.; Ohno, A.; Aida, Y. Bovine leukemia virus proviral load is more strongly associated with bovine major histocompatibility complex class II DRB3 polymorphism than with DQA1 polymorphism in Holstein cow in Japan. Retrovirology 2019, 16, 14. [CrossRef]

32. Takeshima, S.N.; Sasaki, S.; Meripet, P.; Sugimoto, Y.; Aida, Y. Single nucleotide polymorphisms in the bovine MHC region of Japanese Black cattle are associated with bovine leukemia virus proviral load. Retrovirology 2017, 14, 24. [CrossRef]

33. Lo, C.W.; Borjigin, L.; Saito, S.; Fukunaga, K.; Saitou, E.; Okazaki, K.; Mizutani, T.; Wada, S.; Takeshima, S.N.; Aida, Y. BoLA-DRB3 Polymorphism is Associated with Differential Susceptibility to Bovine Leukemia Virus-Induced Lymphoma and Proviral Load. Viruses 2020, 12, 352. [CrossRef] [PubMed]

34. Lutzelschwab, C.M.; Forletti, A.; Cepeda, R.; Esteban, E.N.; Confalonieri, O.; Gutierrez, S.E. Co-infection with Mycobacterium bovis does not alter the response to bovine leukemia virus in BoLA DRB3*0902, genetically resistant cattle. Res. Vet. Sci. 2016, 109, 10-16. [CrossRef]

35. Takeshima, S.N.; Miyasaka, T.; Polat, M.; Kikuya, M.; Matsumoto, Y.; Mingala, C.N.; Villanueva, M.A.; Salces, A.J.; Onuma, M.; Aida, Y. The great diversity of major histocompatibility complex class II genes in Philippine native cattle. Meta Gene 2014, 2, 176-190. [CrossRef] [PubMed]

36. Borjigin, L.; Yoneyama, S.; Saito, S.; Polat, M.; Inokuma, M.; Shinozaki, Y.; Tanaka, N.; Yamanaka, R.; Yasui, A.; Mimura, M.; et al. A novel real time PCR assay for bovine leukemia virus detection using mixed probes and degenerate primers targeting novel BLV strains. J. Virol. Methods 2021, 114264. [CrossRef] [PubMed]

37. Jimba, M.; Takeshima, S.N.; Matoba, K.; Endoh, D.; Aida, Y. BLV-CoCoMo-qPCR: Quantitation of bovine leukemia virus proviral load using the CoCoMo algorithm. Retrovirology 2010, 7, 91. [CrossRef]

38. Takeshima, S.N.; Kitamura-Muramatsu, Y.; Yuan, Y.; Polat, M.; Saito, S.; Aida, Y. BLV-CoCoMo-qPCR-2: Improvements to the BLV-CoCoMo-qPCR assay for bovine leukemia virus by reducing primer degeneracy and constructing an optimal standard curve. Arch. Virol. 2015, 160, 1325-1332. [CrossRef]

39. Sato, H.; Watanuki, S.; Bai, L.; Borjigin, L.; Ishizaki, H.; Matsumoto, Y.; Hachiya, Y.; Sentsui, H.; Aida, Y. A sensitive luminescence syncytium induction assay (LuSIA) based on a reporter plasmid containing a mutation in the glucocorticoid response element in the long terminal repeat U3 region of bovine leukemia virus. Virol. J. 2019, 16, 66. [CrossRef] [PubMed]

40. Bai, L.; Hirose, T.; Assi, W.; Wada, S.; Takeshima, S.N.; Aida, Y. Bovine Leukemia Virus Infection Affects Host Gene Expression Associated with DNA Mismatch Repair. Pathogens 2020, 9, 909. [CrossRef]

41. Assi, W.; Hirose, T.; Wada, S.; Matsuura, R.; Takeshima, S.N.; Aida, Y. PRMT5 Is Required for Bovine Leukemia Virus Infection In Vivo and Regulates BLV Gene Expression, Syncytium Formation, and Glycosylation In Vitro. Viruses 2020, 12, 650. [CrossRef] [PubMed]

42. Komori, H.; Ishiguro, N.; Horiuchi, M.; Shinagawa, M.; Aida, Y. Predominant p53 mutations in enzootic bovine leukemic cell lines. Vet. Immunol. Immunopathol. 1996, 52, 53-63. [CrossRef]

43. Lewin, H.A.; Bernoco, D. Evidence for BoLA-linked resistance and susceptibility to subclinical progression of bovine leukaemia virus infection. Anim. Genet. 1986, 17, 197-207. [CrossRef]

44. Zanotti, M.; Poli, G.; Ponti, W.; Polli, M.; Rocchi, M.; Bolzani, E.; Longeri, M.; Russo, S.; Lewin, H.A.; van Eijk, M.J. Association of BoLA class II haplotypes with subclinical progression of bovine leukaemia virus infection in Holstein-Friesian cattle. Anim. Genet. 1996, 27, 337-341. [PubMed] 
45. Miyasaka, T.; Takeshima, S.N.; Matsumoto, Y.; Kobayashi, N.; Matsuhashi, T.; Miyazaki, Y.; Tanabe, Y.; Ishibashi, K.; Sentsui, H.; Aida, Y. The diversity of bovine MHC class II DRB3 and DQA1 alleles in different herds of Japanese Black and Holstein cattle in Japan. Gene 2011, 472, 42-49. [CrossRef] [PubMed]

46. Xu, A.; van Eijk, M.J.; Park, C.; Lewin, H.A. Polymorphism in BoLA-DRB3 exon 2 correlates with resistance to persistent lymphocytosis caused by bovine leukemia virus. J. Immunol. 1993, 151, 6977-6985.

47. Hayashi, T.; Mekata, H.; Sekiguchi, S.; Kirino, Y.; Mitoma, S.; Honkawa, K.; Horii, Y.; Norimine, J. Cattle with the BoLA class II DRB3*0902 allele have significantly lower bovine leukemia proviral loads. J. Vet. Med. Sci. 2017, 79, 1552-1555. [CrossRef] [PubMed]

48. Agustina, F.; Claudia, M.L.; Rosana, C.; Eduardo, N.E.; Silvina, E.G. Early events following bovine leukaemia virus infection in calves with different alleles of the major histocompatibility complex DRB3 gene. Vet. Res. 2020, 51, 4. [CrossRef]

49. Borjigin, L.; Lo, C.W.; Bai, L.; Hamada, R.; Sato, H.; Yoneyama, S.; Yasui, A.; Yasuda, S.; Yamanaka, R.; Mimura, M.; et al. Risk Assessment of Bovine Major Histocompatibility Complex Class II DRB3 Alleles for Perinatal Transmission of Bovine Leukemia Virus. Pathogens 2021, 10, 502. [CrossRef]

50. Polat, M.; Moe, H.H.; Shimogiri, T.; Moe, K.K.; Takeshima, S.N.; Aida, Y. The molecular epidemiological study of bovine leukemia virus infection in Myanmar cattle. Arch. Virol. 2016. [CrossRef]

51. Panei, C.J.; Takeshima, S.N.; Omori, T.; Nunoya, T.; Davis, W.C.; Ishizaki, H.; Matoba, K.; Aida, Y. Estimation of bovine leukemia virus (BLV) proviral load harbored by lymphocyte subpopulations in BLV-infected cattle at the subclinical stage of enzootic bovine leucosis using BLV-CoCoMo-qPCR. BMC Vet. Res. 2013, 9, 95. [CrossRef] [PubMed]

52. Takeshima, S.N.; Matsumoto, Y.; Miyasaka, T.; Arainga-Ramirez, M.; Saito, H.; Onuma, M.; Aida, Y. A new method for typing bovine major histocompatibility complex class II DRB3 alleles by combining two established PCR sequence-based techniques. Tissue Antigens 2011, 78, 208-213. [CrossRef] [PubMed]

53. Tajima, S.; Aida, Y. Induction of expression of bovine leukemia virus (BLV) in blood taken from BLV-infected cows without removal of plasma. Microbes Infect. 2005, 7, 1211-1216. [CrossRef] [PubMed]

54. Sato, H.; Bai, L.; Borjigin, L.; Aida, Y. Overexpression of bovine leukemia virus receptor SLC7A1/CAT1 enhances cellular susceptibility to BLV infection on luminescence syncytium induction assay (LuSIA). Virol. J. 2020, 17, 57. [CrossRef]

55. Van Der Maaten, M.J.; Miller, J.M. Replication of bovine leukemia virus in monolayer cell cultures. Bibl. Haematol. 1975, 360-362.

56. Onuma, M.; Koyama, H.; Aida, Y.; Okada, K.; Ogawa, Y.; Kirisawa, R.; Kawakami, Y. Establishment of B-cell lines from tumor of enzootic bovine leukosis. Leuk. Res. 1986, 10, 689-695. [CrossRef] 